CHEMICAL GENETICS

\title{
Bumping into AMPK substrates
}

Mol. Cell 44, 878-892 (2011)

AMP-activated protein kinase (AMPK) is generally viewed as a key regulator of energy homeostasis that responds to external stimuli such as nutrient deprivation by phosphorylating protein substrates that reset cellular energy balance. Banko et al. now apply a chemicalgenetic strategy to identify new AMPK substrates and show its involvement in regulating mitotic progression. Mutating the gatekeeper residue of the catalytic domain of AMPK to a glycine residue resulted in an analog-specific variant (AS-AMPK $\alpha 2$ ) that accepts a bulky N6-modified ATP analog as a substrate. AS-AMPK $\alpha 2$ and a thiolated bulky ATP analog were used as reagents in a thiophosphate tagging method that was applied to identify 28 potentially new AMPK targets in $293 \mathrm{~T}$ cells. Several of the candidates were validated as bona fide AMPK substrates in vitro and in cells, and their AMPK $\alpha 2$ phosphorylation sites were mapped by mutational studies. Bioinformatic analysis revealed that many of the hits, including protein phosphatase 1 regulatory subunit 12C (PPP1R12C) and p21-activated protein kinase (PAK2), were proteins associated with mitotic regulation. Further characterization showed that mitotic cells have higher AMPK activities and that inhibition of AMPK activity blocks mitosis in a PPP1R12C phosphorylation-dependent manner. The analog-specific kinase engineering approach revealed a previously unknown role of AMPK in mitotic regulation and may offer a general strategy for identifying cellular substrates of kinases.

\section{METABOLISM}

\section{Closing the loop}

Science, published online 16 December 2011, doi:10.1126/science.1210858

The tricarboxylic acid (TCA) cycle, one of the fundamental biochemical processes in metabolism, generates energy by converting acetate into $\mathrm{CO}_{2}, \mathrm{H}_{2} \mathrm{O}$ and important metabolic precursors. Surprisingly, cyanobacteria do not have a copy of 2-oxoglutarate dehydrogenase (2-OGDH), one of the traditional TCA enzymes, spurring alternate theories about how these species meet their metabolic needs. Mycobacterium spp. are known to use 2-oxoglutarate decarboxylase (2-OGDC) in tandem with succinic semialdehyde dehydrogenase (SSADH) as an alternative to 2-OGDH, but cyanobacteria also lack any obvious homologs of 2-OGDC. However, cyanobacteria do encode an SSADH homolog, suggesting to Zhang and Bryant that an unrecognized version of the decarboxylase might also exist. Gene neighborhood analysis of Synechococcus sp. PCC 7002 identified a two-gene operon encoding SSADH and a second putative enzyme with the same cofactor dependence as the other 2-oxoglutarate-converting enzymes. Analysis of purified proteins showed that the putative 2-OGDC produced an aldehyde, as expected, and a coupled assay confirmed the conversion of 2-oxoglutarate to succinate. Cellular assays indicated that the enzymes were present, functional and required for normal growth. Bioinformatics identified the new gene in numerous species, suggesting that this alternate solution is widely adopted. Though it remains to be seen how the two
2-OGDC enzymes are divergent in sequence yet overlapping in function, this result should initiate a substantial rethinking of cyanobacterial metabolism.

TRANSLATION

\section{Ribosome 3.0}

Science 334, 1524-1529 (2011)

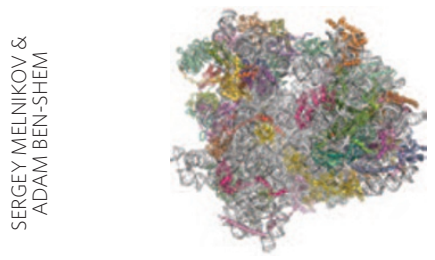

Although the translation of mRNA to protein by ribosomes is generally conserved, there are ribosomal components specific to eukaryotes. Ben-Shem et al. now report the first atomic model of the $80 \mathrm{~S}$ ribosome from Saccharomyces cerevisiae based on X-ray crystal data resolved to 3.0 ̊. Their model includes two ribosomes in different conformations containing all 35 universally conserved proteins as well as the 44 eukaryotespecific ones. The latter components as well as eukaryote-specific rRNA are predominately located on the surface of the ribosome but away from the regions responsible for executing core ribosomal activities. The authors also noted that unlike in prokaryotic rRNAs, nonhelical elements of rRNA formed singlestranded stretches that serve as platforms for protein binding. The interface between the subunits was approximately doubled in eukaryotes, owing to the formation of more intersubunit bridges. Indeed, two bridges created by eukaryote-specific extensions were not buried between the subunits but were solvent exposed. Their location with respect to other known functional landmarks led the authors to hypothesize that they have a role in translational initiation. The structure also included a nonribosomal protein, the translational inhibitor Stm1, which appeared to block the mRNA access tunnel via the insertion of an $\alpha$-helix that mimics the path of the mRNA to the active site of the ribosome. Although hypotheses resulting from this structure remain to be experimentally validated, this structure provides the first molecular-level model of the complete eukaryotic ribosome.

MOLECULAR SWITCHES

\section{The pore of the matter PLoS Pathog. 7, e1002354 (2011)}
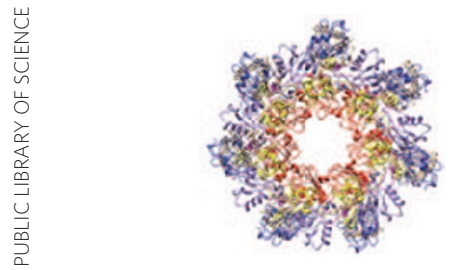

The entry of anthrax toxin into host cells after Bacillus anthracis infection follows a series of well-known steps, from binding of the $83-\mathrm{kDa}$ toxin $\left(\mathrm{PA}_{83}\right)$ to cellular receptors such as ANTXR2 and cleavage to and heptamerization of a 63-kDa form $\left(\mathrm{PA}_{63}\right)$ that forms a 'prepore' structure, to formation of a $\mathrm{PA}_{63}$ pore within acidic compartments that allow the toxin to escape into the host cytosol. It is thought that binding of the toxin to the VWA domain of ANTXR2 hinders movements that are necessary for pore formation; once this interaction is released by the low $\mathrm{pH}$ of internal compartments, the pore can readily form. To learn more about the PA-receptor interaction, such as whether the complex dissociates within cells, Pilpa et al. used the NMR transfer cross-saturation method, which can be used to identify the binding interface of large protein-protein complexes. The data comparing contacts at $\mathrm{pH}$ 5.1, 6.0 and 8.0 suggest a model in which the receptor remains bound to domain 4 of PA but loses its interactions with PA domain 2 prior to prepore-to-pore conversion. The partially bound receptor may act to stabilize the toxin heptameric structure. They also reveal a new toxin-receptor intermediate, which possibly exists in the mildly acidic early endosome, on the path to pore formation.

Written by Mirella Bucci, Amy Donner, Joshua M. Finkelstein, Catherine Goodman \& Terry L. Sheppard 